\title{
Efficient Algorithm to Compute PDEs on the Grid
}

\author{
Marc Garbey \\ University of Houston
}

\begin{abstract}
This presentation will discuss the design of numerically efficient algorithm to solve PDEs on the Grid. Efficiency of numerical algorithms are strongly dependent on the features of computer architecture. In western countries the architecture of large scale parallel computer is essentially driven by industrial cost. Large "Uniform" Multi-Processors Architecture are replaced progressively by Multiclusters Architecture or Beowulf systems that are cheaper. In such architecture Communication and/or memory access is generally very slow compare to the speed of the cpu units. Further cpu units may have several levels of cache and memory. It is then very difficult to achieve peak performance even on a single computer. The situation does not seems to improve: cpu flops still increases by a factor 2 every 18 months while bandwidth to access the memory is improving very slowly. An extreme situation is grid computing for which networks are at least one or two order of magnitude slower than internal network of parallel architecture. It is therefore a general problem to design numerical algorithm that can keep parallel efficiency in such environment. Meta-computing is a good test bed because it is probably the most challenging situation to achieve efficiency of a numerical scheme. Any numerical algorithm that will be efficient on Metacomputing Architecture such as a grid of parallel computers linked by a slow network should be of interest for large ASCI machine as well. There are some simple general idea that one may keep in mind to design numerical algorithm for PDE applications in grid environment: - ForWave propagation phenomenon: Speed of propagation of data in Memory should be the analogue of the speed of propagation of waves. - For Heat Transfer: Domain of influence of data in memory should be set according to decay of heat in space, i.e finite size depending on the level of accuracy. - For Steady flow: Data representation and space dependencies should match the memory Hierarchy. - For data transfer in Network, one may split the significant digits corresponding to required accuracy from the digits required for
\end{abstract}


stability only. As a matter of fact in most of the applications, one look for a numerical result within one per cent accuracy and the need to carry all digits in communications should be review according to stability theory. In this paper we will concentrate on domain decomposition methods that are particularly efficient for large scale metacomputing. 